\title{
Simulation Study of Balancing Arm Arrangement for Controlling Base Disturbances in Long Reach Manipulators
}

\author{
A. SRINIVASAN*, A. JEGAN** \\ *Anna Universtiy, Chennai, Tamilnadu, India, E-mail: ramanasai2019@gmail.com \\ **Department of Mechanical Engineering, Sona College of Technology, Salem, Tamilnadu, India, \\ E-mail: jegmak@gmail.com \\ crossref http://dx.doi.org/10.5755/j02.mech.27493
}

\section{Introduction}

Many terrestrial and space applications require remotely operated tasks. Some such examples are repair of bridges, handling of nuclear wastes, aerial refuelling, maintenance of international space station, and payload tending in space $[1,2]$. Long Reach Manipulator (LRM) system is inevitable for such applications. The LRM is a long multi-link serial chain carrying a Short Reach Arm (SRM) at its free end as end-effector. The LRM performs gross motion by deploying the SRM at the required task location; while the SRM performs fine motion for accomplishing the mission task. The dynamic coupling between the LRM and its supporting base causes undesirable motion of the base, leading to improper location and orientation of the SRM [3]. The main issue of such disturbance is the change in base attitude that shall severe the signals from ground stations; the position and attitude change shall also create collision of the SRM while performing the mission task [4]. Hence base disturbance of LRM system has been remaining as an active research area.

In their extensive survey, Dwivedi and Eberhard [5] discussed the modelling, control, and experiments on the dynamics of flexible manipulators, and their varied applications. Dubowsky and Papadopoulos [6] identified and discussed some important dynamics and control problems that arise in free-flying and free-floating space robotics. Early researches on vibration problems of space manipulators suggested that effective methods of modelling, control and planning the motion of the manipulators must be attempted to reduce the amount of vibration during manoeuvre of the arm [7]. Studies had been carried out for suggesting the specific motion of the rigid SRM that will transmit a prescribed force to the supporting flexible arm to arrest the vibration effects [8]; a new active damping algorithm by using the reaction force from the SRM on the flexible LRM as a control variable [9]; a novel concept of Dynamically Equivalent Manipulator which could be built easily for realistic experiments in the laboratory, and used as a tool for dynamic modeling of space manipulators for reduced gravity situations [10]. George and Book [11] suggested a control scheme for inertial vibration damping of a flexible base manipulator by making the link accelerations out of phase with the base velocity, and removing the vibration from the system. Zhu et al. [12] performed an analytical study, on the nonlinear dynamics of a two degree of freedom (DoF) vibration system, incorporating nonlinear damping and nonlinear springs, and reported that the reduction in amplitude and oscillations could be obtained by suitably adjusting the system parameters with proper excitation frequency. Chalfoun et al. [13] designed and analyzed a special parallelogram linkage with glass epoxy springs for gravity compensation in long multi-link carrier for nuclear remediation. Özer and Semercigil [14] experimented the variable stiffness control technique for controlling the excessive oscillations in a twolink flexible robotic arm and reported that the method was stable and relatively insensitive to significant parameter changes. Cocuzza et al. [15] developed a novel theoretical formulation for the inverse kinematics of redundant space robotic systems for locally minimizing the torque transferred to the spacecraft due to the robotic arm movement. Experimental validation through tests on a $2 \mathrm{D}$ robot, under simulated microgravity conditions, suggested the suitability of the proposed solution. Nenchev [16] developed the Reaction Null Space formalism and suggested the implementation of the same for base disturbance of free floating space robot. Minghe et al. [17] considered the reaction torque acting on the satellite base as an important index to measure the base disturbance, and proposed a novel way to derive the analytical form of the reaction torque. Through empirical results they suggested that the analysis about reaction torque was valid and that the proposed method was suitable for base disturbance compensation.

Van der Wijk et al. [18] compared and evaluated, numerically and analytically, the different principles of dynamic balancing. They made a detailed literature survey, identified the balancing principles, and classified them as deploying counter-rotary counter masses, counter masses and separate counter rotations, and duplicate mechanisms in the form of balance links. The authors concluded that when both mass and inertia addition are important, the duplicate mechanism method is the most suitable one for low-mass and low-inertia dynamic balancing, which is a principle used for balancing the mechanism altogether. The balancing link principle has been taken for analysis in this work.

In literature, a very few work has been reported on the design of augmented mechanisms, in the form of balancing links, that can compensate base disturbances in LRM systems. A balancing arm performs controlled redundant motion for controlling the base disturbances. Hence, the mass of the balancing-arm system is to be kept lesser in order to reduce the driving torque. In space applications, this will also address the demands on space fuel that has a need to be economized. Huang et al. [4] considered the analysis of multi-arm space manipulators, of which one arm is the mission arm, while all the others were the balancing arms. Also, for simulation study, they took two arms each with three links, one acting as the main arm 
and the other as balancing arm, and for simplifying the problems, they considered both the arms to be of same geometric structure, and hence same mass. This paper has considered the balancing arm of different geometric structure for keeping its mass reduced as compared to that of the main arm. A planar arrangement of a two-link mission arm with payload, and a one-link balance arm with a tip mass is modelled for analysis. The tip mass has the provision for keeping it constant or dynamically varying. The bounce and pitch of the base, arising out of reaction force and torque, are obtained separately for the constant and variable mass conditions of compensating arm, and the results are correspondingly compared to analyse the effect of the mass of balancing arm. The mission arm or the LRM system is referred as main arm and the balance arm, as compensating arm.

The paper is presented in the following sections: Section 2 explains the modelling of the manipulator and the compensating arm arrangement, Section 3 explains the mathematical formulation, Section 4 highlights the SIMULINK subsystems, Section 5 explains the simulation procedure, Section 6 presents an analysis and discussion of the simulation results, and Section 7 draws the conclusions.

\section{Modelling of the manipulator and compensating arm}

The main arm is modelled as a serial kinematic chain with two links. The first link is longer and it idealizes the manipulator arm. One end of this link is pivoted to the base and the other end is pivoted to the second link. The second link is of shorter length, and it carries the payload at its free end. This link models the SRM. The compensating arm is a single link, one end of which is pivoted to the base, while the other end carries the tip mass. The base supports both the arms, and for the purpose of study, it is assumed to be mounted on linear elastic springs. Fig. 1 illustrates the LRM and the compensating arm arrangement.

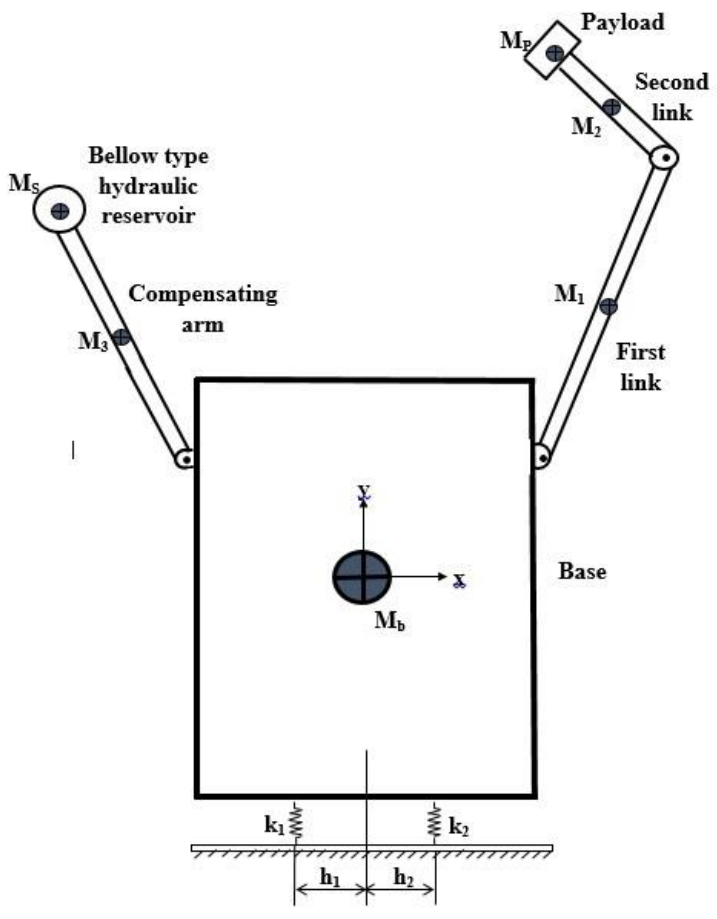

Fig. 1 Model of the manipulator and the compensating arm arrangement
Since the compensating arm is an appendage to the main LRM system, its inertial forces are undesirable and hence its mass has to be kept minimum. As shown in Fig. 2, the tip mass of the compensating arm is incorporated as a bellows-like hydraulic reservoir which receives, stores, and expels fluid. The hydraulic control unit, housed at the base, controls the variation of the volume of fluid in the reservoir commensurate with the movement of the main arm. Assuming the density of the fluid to be constant, the tip mass in the compensating arm is thus dynamically varied.

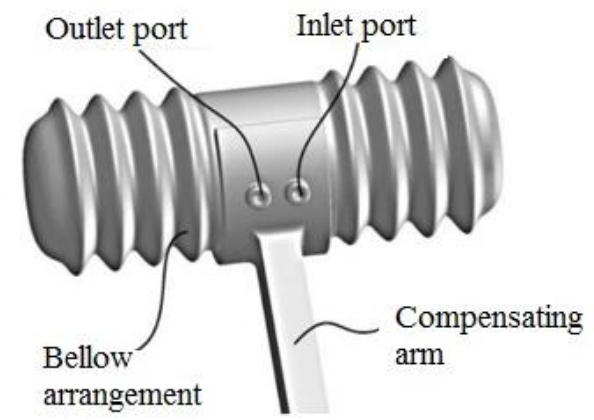

Fig. 2 Bellows-like hydraulic reservoir as tip mass

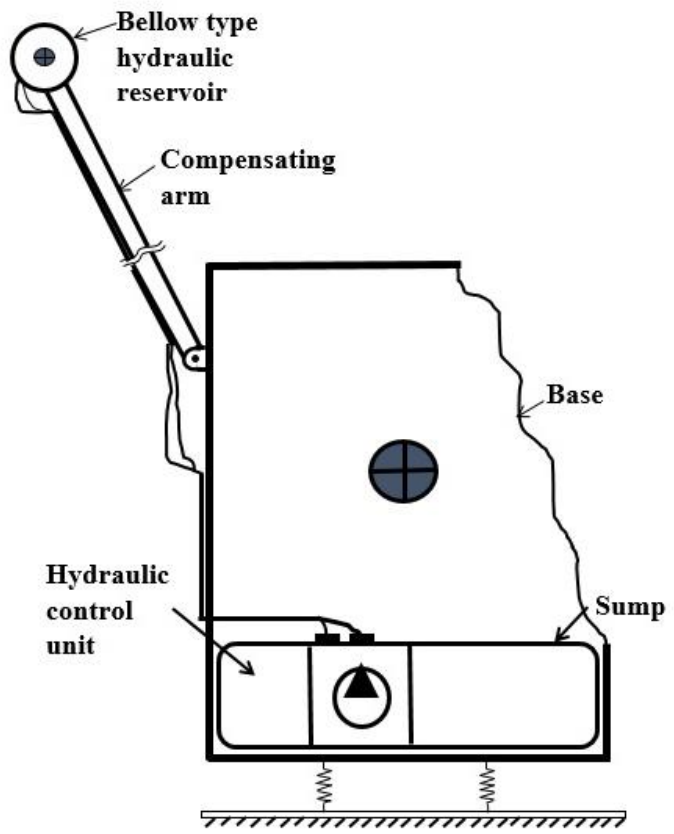

Fig. 3 Schematic of hydraulic control

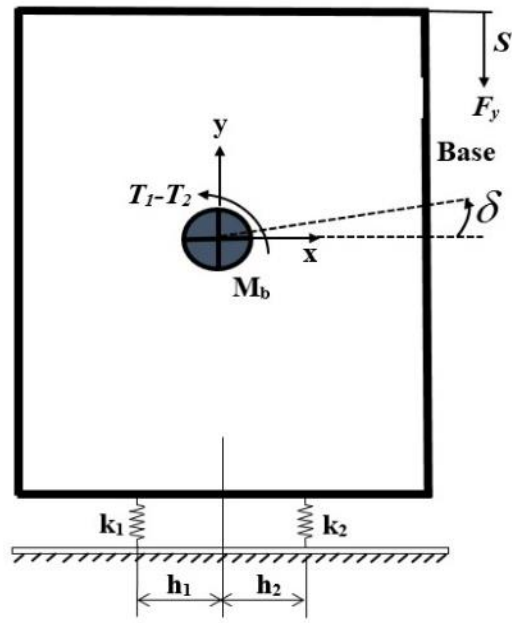

Fig. 4 Base modelled as half car 
Fig. 3 illustrates the schematic of the hydraulic control unit. The control unit senses the disturbing torque on the base and correspondingly controls the flow of fluid in the circuit connecting the reservoir at the tip. The hydraulic control unit comprises the sump, separate pumps for charging and discharging the fluid into the bellows or out of it, and direction control valves for controlling the direction of fluid flow. As shown in Fig. 4, the base is modelled as a half car and the disturbance is analysed as the bounce and pitch motion of the base.

\section{Mathematical formulation}

\subsection{Simultaneous constraint method}

The simultaneous constraint matrix is developed with the help of the equations from the vector loop connecting the links, and the free body diagram of each link [19]. The rectangular components of the second derivate of the kinematic equations from the vector loops contain the unknown quantities of angular velocities and angular accelerations of the links. The external forces and unknown joint forces are represented on the free body diagram of each link. The force balance equations, written using the NewtonEuler formulation, relate these forces with the link's acceleration. Further, treating the links as vector chains, additional information about the acceleration of the centre of mass (COM) of each link are obtained. This system of equations is written as a matrix equation in the form:

$$
[A]\{x\}=\{b\},
$$

where: $[A]$ is a sparse matrix containing the parameters of the links; $\{x\}$ is the vector of unknown quantities like kinematic parameters and joint forces; $\{b\}$ is the vector of known quantities. The above matrix is solved using MATLAB and SIMULINK as a full dynamic simulation of the system.

\subsection{Dynamic equations of the model}

The assumptions made in the derivation are: the links and the base are considered as rigid bodies; the fluid flow is steady and flow paths are non-constricted and hence inertance is negligible; the control by the hydraulic circuit is effected instantaneously, and the change in the position vector of COM of compensating arm due to tip mass variation is negligible.

Fig. 5 shows the model configuration indicating the various position vectors and joint angles. The COM of the base is taken as the reference frame. The nomenclature used are: ro, rs1 - distance of pivots of first link and compensating link from reference frame; $r_{1}, r_{2}, l_{s 1}-$ lengths of first, second and compensating links; $\mathbf{r}_{\mathbf{p} 1}, \mathbf{r}_{\mathbf{p} 2}, \mathbf{r}_{\mathbf{p} 3}, \mathbf{r}_{\mathbf{s} 2}, \mathbf{r}_{\mathbf{s} 3}$ - position vectors of COM of links, payload and tip mass; $M_{b}, M_{1}, M_{2}, M_{P}-$ mass of base, first, second link and payload; $M_{3}, M_{\mathrm{S}}$ - mass of compensating link, tip mass; $\phi_{0}$ and $\beta_{0}$ - angle of pivot of first and compensating link from horizontal; $\phi_{1}, \phi_{2}, \beta_{2}$ - orientation of first, second and compensating links.

For the main arm, the position equations of the first link, second link and payload are written. By differentiating the position equations twice, the acceleration equations are obtained as below:
Using the short form:

$$
\begin{aligned}
& \cos \left(\phi_{0}\right)=C_{0}, \sin \left(\phi_{0}\right)=S_{0} \\
& \cos \left(\phi_{0}+\phi_{1}\right)=C_{01}, \sin \left(\phi_{0}+\phi_{1}\right)=S_{01} \\
& \cos \left(\phi_{0}+\phi_{1}+\phi_{2}\right)=C_{012}, \sin \left(\phi_{0}+\phi_{1}+\phi_{2}\right)=S_{012}
\end{aligned},
$$

the components of acceleration of first link, second link and payload are obtained as given in Eqs. (2-4).

$$
\begin{aligned}
& \ddot{r}_{p 1 x}=-l_{c 1} S_{01} \alpha_{1}-l_{c 1} C_{01} \omega_{1}^{2} \\
& \ddot{r}_{p 1 y}=l_{c 1} C_{01} \alpha_{1}-l_{c 1} S_{01} \omega_{1}^{2}, \\
& \ddot{r}_{p 2 x}=-\left(r_{1} S_{01}+l_{c 2} S_{012}\right) \alpha_{1}-l_{c 2} S_{012} \alpha_{2}- \\
& -\left(l_{c 2} C_{012}+r_{1} C_{01}\right) \omega_{1}^{2}-l_{c 2} C_{012} \omega_{2}^{2}-2 l_{c 2} C_{012} \omega_{1} \omega_{2}, \\
& \ddot{r}_{p 2 y}=\left(r_{1} C_{01}+l_{c 2} C_{012}\right) \alpha_{1}+l_{c 2} C_{012} \alpha_{2}- \\
& -\left(l_{c 2} S_{012}+r_{1} S_{01}\right) \omega_{1}^{2}-l_{c 2} S_{012} \omega_{2}^{2}-2 l_{c 2} S_{012} \omega_{1} \omega_{2} \\
& \ddot{r}_{p 3 x}=-\left(r_{1} S_{01}+r_{2} S_{012}\right) \alpha_{1}-r_{2} S_{012} \alpha_{2}- \\
& -\left(r_{2} C_{012}+r_{1} C_{01}\right) \omega_{1}^{2}-r_{2} C_{012} \omega_{2}^{2}-2 r_{2} C_{012} \omega_{1} \omega_{2} . \\
& \ddot{r}_{p 3 y}=\left(r_{1} C_{01}+r_{2} C_{012}\right) \alpha_{1}+r_{2} C_{012} \alpha_{2}- \\
& -\left(r_{2} S_{012}+r_{1} S_{01}\right) \omega_{1}^{2}-r_{2} S_{012} \omega_{2}^{2}-2 r_{2} S_{012} \omega_{1} \omega_{2}
\end{aligned}
$$

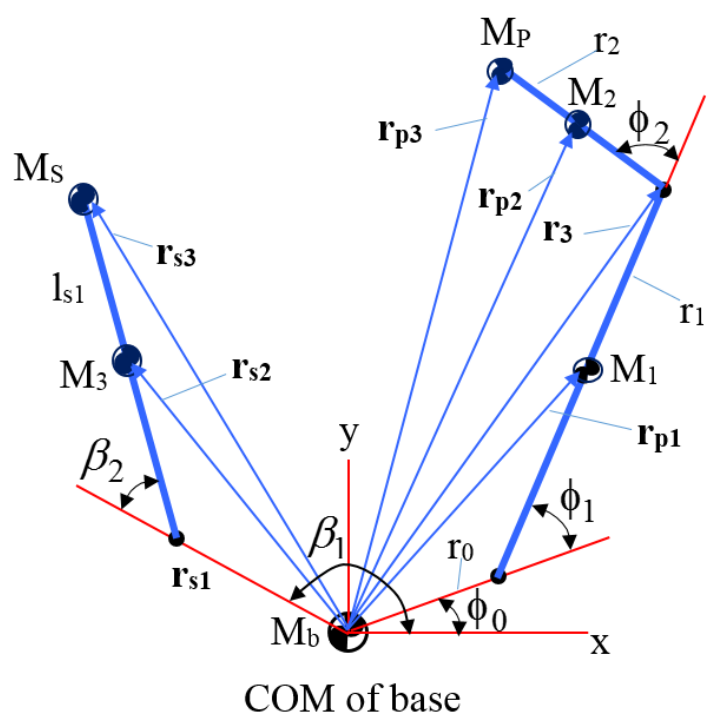

Fig. 5 Model configuration

The free body diagrams of the first link, second link along with payload are drawn as in Fig. 6.
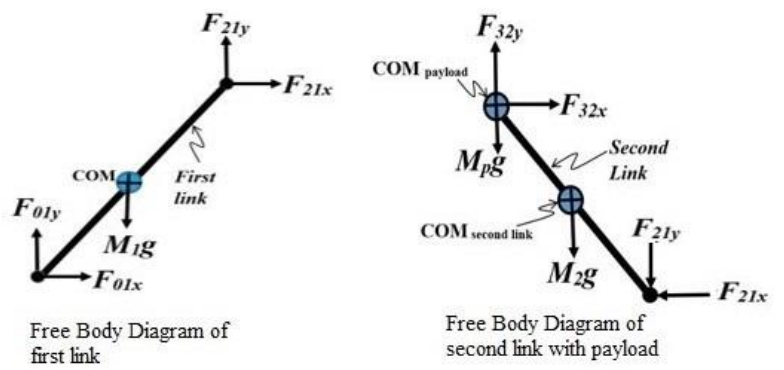

Fig. 6 Free Body Diagrams of first and second link

Using Newton-Euler equations, the force balance equation for first link, second link, and payload are given in Eqs. $(5,6,7)$ respectively.

$$
\begin{aligned}
& F_{01 x}+F_{21 x}=M_{1} \ddot{r}_{p 1 x} \\
& F_{01 y}+F_{21 y}-M_{1} g=M_{1} \ddot{r}_{p 1 y},
\end{aligned}
$$




$$
\begin{aligned}
& -F_{21 x}+F_{32 x}=M_{2} \ddot{r}_{p 2 x} \\
& -F_{21 y}+F_{32 y}-M_{2} g=M_{2} \ddot{r}_{p 2 y} \text {, } \\
& -F_{32 x}=M_{p} \ddot{r}_{p 3 x} \\
& -F_{32 y}-M_{p} g=M_{p} \ddot{r}_{p 3 y} \\
& \begin{array}{l}
T_{1}=F_{01 x} r_{0 y}-F_{01 y} r_{0 x}+F_{21 x} r_{3 y}-F_{21 y} r_{3 x}+F_{32 x} r_{p 3 y}-F_{32 y} r_{p 3 x}+M_{1} \ddot{r}_{p 1 x} r_{p 1 y}-M_{1} \ddot{r}_{p 1 y} r_{p 1 x}+ \\
+M_{2} \ddot{r}_{p 2 x} r_{p 2 y}-M_{2} \ddot{r}_{p 2 y} r_{p 2 x}+M_{p} \ddot{r}_{p 3 x} r_{p 3 y}-M_{p} \ddot{r}_{p 3 y} r_{p 3 x}
\end{array} .
\end{aligned}
$$

Using the Eqs. (2-8), the simultaneous constraint matrix for main arm is written as shown in Eq. (9). Using a similar procedure, the simultaneous constraint matrix for
The disturbing torque at the COM of the base about $\mathrm{z}$-axis is obtained using D'Alembert's principle and written by adding the products of all forces with their respective moment arms. Thus the disturbing torque due to LRM

$$
A=\left[\begin{array}{ccccccccccccc}
1 & 0 & 0 & 0 & 0 & 0 & 0 & 0 & 0 & 0 & 0 & 0 & 0 \\
0 & 1 & 0 & 0 & 0 & 0 & 0 & 0 & 0 & 0 & 0 & 0 & 0 \\
0 & 0 & 1 & 0 & 0 & 0 & 0 & 0 & 0 & 0 & 0 & 0 & 0 \\
0 & 0 & 0 & 1 & 0 & 0 & 0 & 0 & 0 & 0 & 0 & 0 & 0 \\
0 & 0 & 0 & 0 & 1 & 0 & 0 & 0 & 0 & 0 & 0 & 0 & 0 \\
0 & 0 & 0 & 0 & 0 & 1 & 0 & 0 & 0 & 0 & 0 & 0 & 0 \\
-M_{1} & 0 & 0 & 0 & 0 & 0 & 1 & 0 & 1 & 0 & 0 & 0 & 0 \\
0 & -M_{1} & 0 & 0 & 0 & 0 & 0 & 1 & 0 & 1 & 0 & 0 & 0 \\
0 & 0 & -M_{2} & 0 & 0 & 0 & 0 & 0 & -1 & 0 & 1 & 0 & 0 \\
0 & 0 & 0 & -M_{2} & 0 & 0 & 0 & 0 & 0 & -1 & 0 & 1 & 0 \\
0 & 0 & 0 & 0 & M_{P} & 0 & 0 & 0 & 0 & 0 & 1 & 0 & 0 \\
0 & 0 & 0 & 0 & 0 & M_{P} & 0 & 0 & 0 & 0 & 0 & 1 & 0 \\
M_{1} r_{p 1 y} & -M_{1} r_{p 1 x} & M_{2} r_{p 2 y} & -M_{2} r_{p 2 x} & M_{P} r_{p 3 y} & -M_{P} r_{p 3 x} & r_{0 y} & -r_{0 x} & r_{3 y} & -r_{3 x} & r_{p 3 y} & -r_{p 3 x} & 1
\end{array}\right] ;
$$$$
x=\left[\begin{array}{c}
\ddot{r}_{p 1 x} \\
\ddot{r}_{p 1 y} \\
\ddot{r}_{p 2 x} \\
\ddot{r}_{p 2 y} \\
\ddot{r}_{p 3 x} \\
\ddot{r}_{p 3 y} \\
F_{01 x} \\
F_{01 y} \\
F_{21 x} \\
F_{21 y} \\
F_{32 x} \\
F_{32 y} \\
T_{1}
\end{array}\right] ; B=\left[\begin{array}{c}
-l_{c 1} C_{01} \omega_{1}^{2}-l_{c 1} S_{01} \alpha_{1} \\
-l_{c 1} S_{01} \omega_{1}^{2}+l_{c 1} C_{011} \alpha_{1} \\
-\left(r_{1} S_{01}+l_{c 2} S_{012}\right) \alpha_{1}-l_{c 2} S_{012} \alpha_{2}-\left(r_{1} C_{01}+l_{c 2} C_{012}\right) \omega_{1}^{2}-l_{c 2} C_{012} \omega_{2}^{2}-2 l_{c 2} C_{012} \omega_{1} \omega_{2} \\
\left(r_{1} C_{01}+l_{c 2} C_{012}\right) \alpha_{1}+l_{c 2} C_{012} \alpha_{2}-\left(r_{1} S_{01}+l_{c 2} S_{012}\right) \omega_{1}^{2}-l_{c 2} S_{012} \omega_{2}^{2}-2 l_{c 2} S_{012} \omega_{1} \omega_{2} \\
-\left(r_{1} S_{01}+r_{2} S_{012}\right) \alpha_{1}-r_{2} S_{012} \alpha_{2}-\left(r_{1} C_{01}+r_{2} C_{012}\right) \omega_{1}^{2}-r_{2} C_{012} \omega_{2}^{2}-2 r_{2} C_{012} \omega_{1} \omega_{2} \\
\left(r_{1} C_{01}+r_{2} C_{012}\right) \alpha_{1}+r_{2} C_{012} \alpha_{2}-\left(r_{1} S_{01}+r_{2} S_{012}\right) \omega_{1}^{2}-r_{2} S_{012} \omega_{2}^{2}-2 r_{2} S_{012} \omega_{1} \omega_{2} \\
0 \\
M_{1} g \\
0 \\
M_{2} g \\
0 \\
-M_{p} g \\
M_{1} g r_{p 1 x}+M_{2} g r_{p 2 x}
\end{array}\right]
$$

$$
\left[\begin{array}{ccccccc}
1 & 0 & 0 & 0 & 0 & 0 & 0 \\
0 & 1 & 0 & 0 & 0 & 0 & 0 \\
0 & 0 & 1 & 0 & 0 & 0 & 0 \\
0 & 0 & 0 & 1 & 0 & 0 & 0 \\
-M_{3} & 0 & -M_{S} & 0 & 1 & 0 & 0 \\
0 & -M_{3} & 0 & -M_{S} & 0 & 1 & 0 \\
M_{3} r_{s 2 y} & M_{3} r_{s 2 x} & M_{3} r_{s 3 y} & M_{3} r_{s 3 x} & -r_{s 1 y} & -r_{s 1 x} & 1
\end{array}\right]\left[\begin{array}{c}
\ddot{r}_{s 2 x} \\
\ddot{s}_{s 2 y} \\
\ddot{r}_{s 3 x} \\
\ddot{r}_{s 3 y} \\
F_{45 x} \\
F_{45 y} \\
T_{2}
\end{array}\right]=\left[\begin{array}{c}
r_{s 1} C_{1} \dot{\beta}_{1}^{2}+r_{s 1} S_{1} \ddot{\beta}_{1}+l_{s c 1} C_{12} \dot{\beta}^{2}+l_{s c 1} S_{12} \ddot{\beta} \\
-r_{s 1} S_{1} \dot{\beta}_{1}^{2}+r_{s 1} C_{1} \ddot{\beta}_{1}-l_{s c 2} S_{12} \dot{\beta}^{2}+l_{s c} C_{12} \ddot{\beta} \\
r_{s 1} C_{1} \dot{\beta}_{1}^{2}+r_{s 1} S_{1} \ddot{\beta}_{1}+l_{s 2} C_{12} \dot{\beta}^{2}+l_{s 2} S_{12} \ddot{\beta} \\
-r_{s 1} S_{1} \dot{\beta}_{1}^{2}+r_{s 1} C_{1} \ddot{\beta}_{1}-l_{s 2} S_{12} \dot{\beta}^{2}+l_{s 2} C_{12} \ddot{\beta} \\
0 \\
M_{3} g+M_{S} g \\
-M_{3} g r_{s 2 x}-M_{S} g r_{s 3 x}
\end{array}\right] .
$$




$$
\left[\begin{array}{cc}
\left(k_{1}+k_{2}\right) & \left(k_{1} h_{1}-k_{2} h_{2}\right) \\
\left(k_{1} h_{1}-k_{2} h_{2}\right) & \left(k_{1} h_{1}^{2}-k_{2} h_{2}^{2}\right)
\end{array}\right]\left[\begin{array}{l}
S \\
\delta
\end{array}\right]=\left[\begin{array}{c}
F_{y} \\
\left(T_{1}-T_{2}\right)
\end{array}\right] \text {. }
$$

The following short forms have been used in compensating arm constraint matrix:

$$
\begin{aligned}
& \cos \left(\beta_{1}\right)=C_{1}, \sin \left(\beta_{1}\right)=S_{1} \\
& \cos \left(\beta_{1}+\beta_{2}\right)=C_{12}, \sin \left(\beta_{1}+\beta_{2}\right)=S_{12} \\
& \beta=\left(\beta_{1}+\beta_{2}\right), \dot{\beta}^{2}=\left(\dot{\beta}_{1}+\dot{\beta}_{2}\right)^{2}, \ddot{\beta}=\left(\ddot{\beta}_{1}+\ddot{\beta}_{2}\right)
\end{aligned},
$$

where: $S$ and $\delta$ in base equations represent the bounce and pitch motions.

\section{Simulink models}

The different SIMULINK models developed and their interaction is illustrated in Fig. 7.

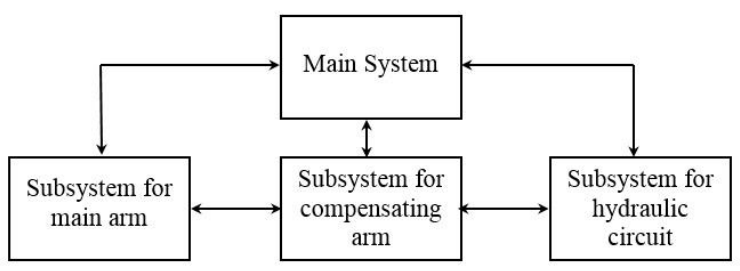

Fig. 7 Simulink subsystems

Apart from integrating the three subsystems, the main system also computes the pitch and bounce of the base. The subsystem for main arm takes the link angles as initial conditions and computes the angular velocities and angular accelerations. Further this system computes the position vectors and feeds, through the MUX block, to function file that computes and outputs the joint forces, linear accelerations and torque about the COM of the base. The subsystem for compensating arm takes the torque of the main arm as input. The function file in this system computes the linear accelerations, joint forces and torque due to the movement of the compensating arm. This torque is added to the main arm input torque to produce the error torque signal. This signal is fed to the PI controller which senses this signal and accordingly actuates the compensating arm, thus drifting the error torque towards zero. The compensating arm subsystem can be simulated in two conditions: keeping the tip mass as fixed quantity, and varying the tip mass. The variation in the tip mass is enabled by the subsystem for hydraulic circuit. This subsystem takes error torque as input signal and compares with set point (set as zero, corresponding to equilibrium position), and depending on the difference, the detect-increase or detect-decrease function blocks drive logic gates to operate the corresponding direction control valve to supply into or expel out fluid from the bellows. The magnitude of the torque is the product of the force due to the mass and the moment arm. Thus here the mass is dynamically varied in order to control the error torque.

\section{Simulation procedure}

The execution flow in the subsystems are illustrated in the Figs. 8 and 9.

\section{Subsystem for main arm}

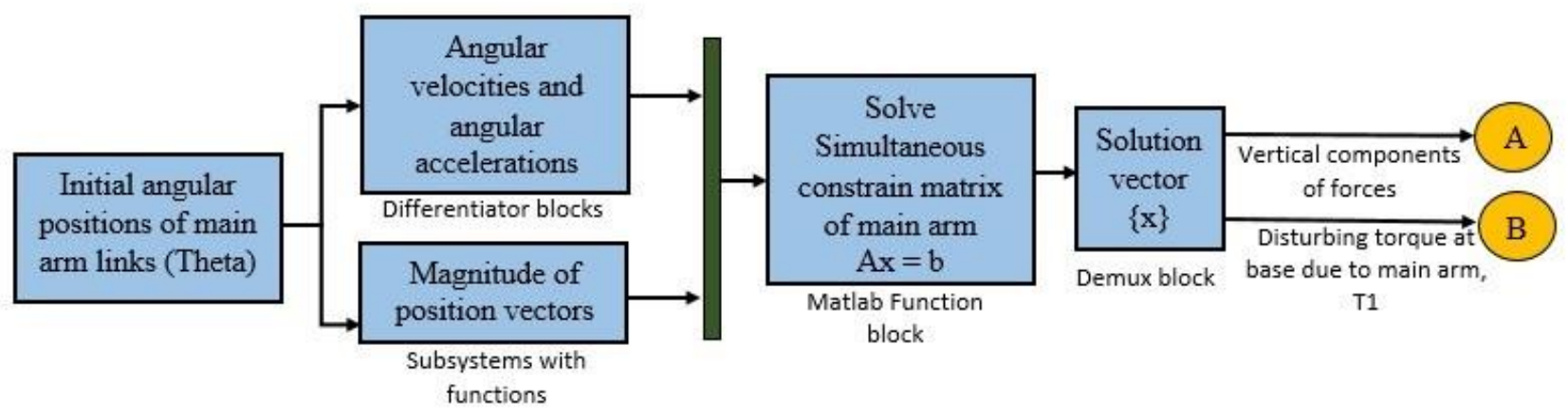

Subsystem for compensating arm

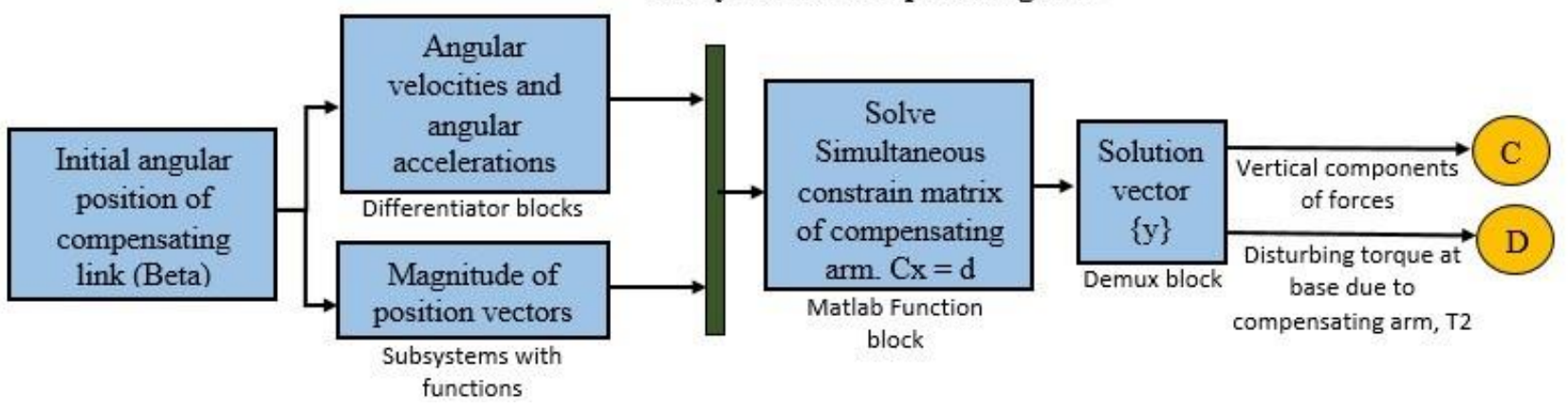

Fig. 8 Simulink execution flow for main arm and compensating arm subsystems 


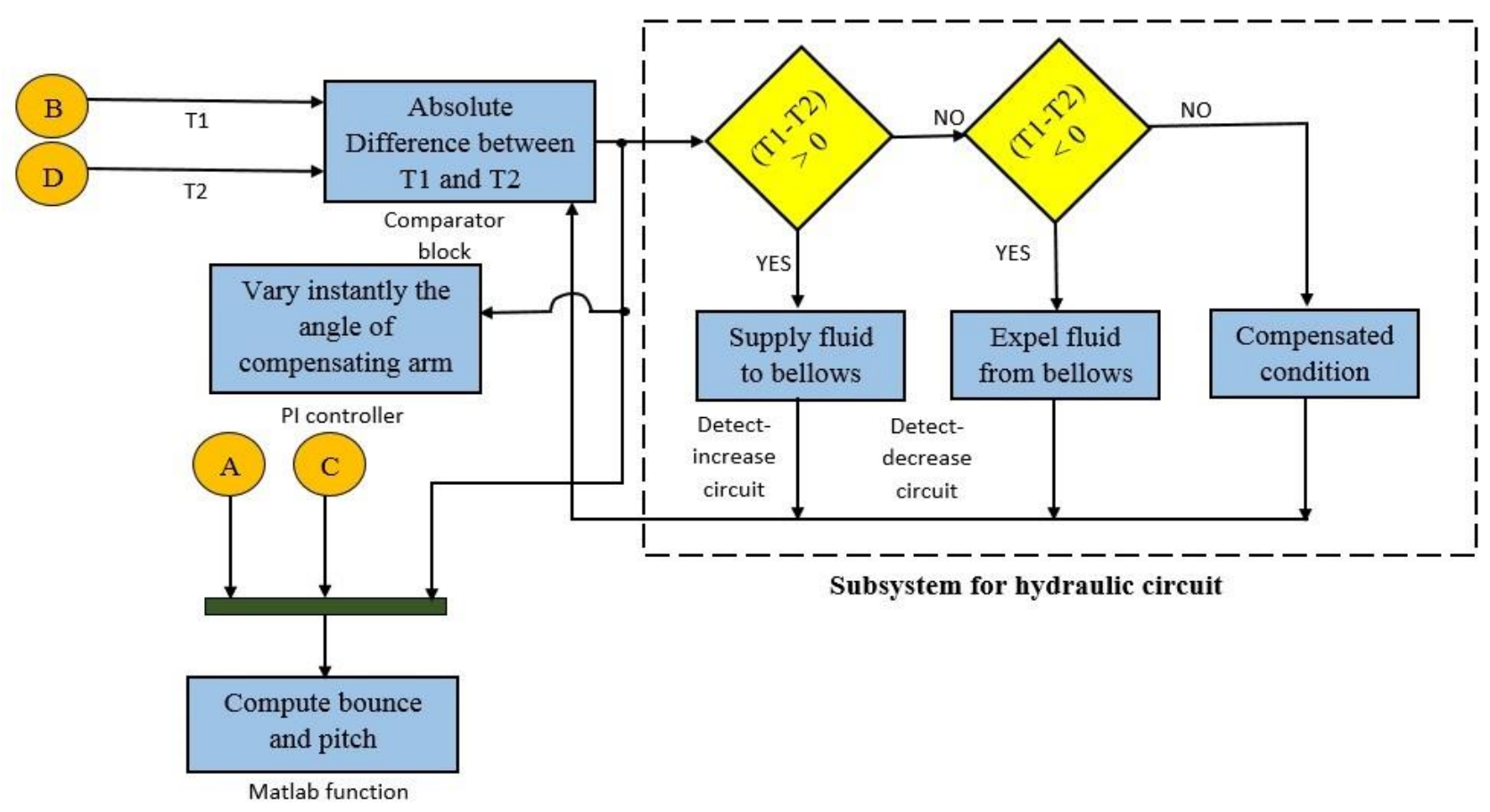

Fig. 9 Simulink execution flow for hydraulic subsystem

The parameters and constants chosen for simulation are indicated in Table 1 . The values are so chosen as to enable an experimental validation as a logical extension of this study. The mass properties are obtained by constructing solid models as per dimensions. The spring constants are chosen arbitrarily to observe base movements conveniently as pitch and bounce. The link parameters are input to the MATLAB workspace to be available for the function blocks, and the initial conditions are fed through appropriate dialog boxes. The main arm links are actuated through $50^{\circ}$ by ramp signal. Simulation is performed in the two conditions of keeping the tip mass constant and varying the tip mass. The error torque, mass of fluid in the reservoir, pitch and bounce of the base are obtained from the simulation.

Table 1

Parameters for simulation

\begin{tabular}{|c|c|c|c|c|c|c|}
\hline \multirow{2}{*}{ Parameter } & \multirow{2}{*}{ Base } & \multicolumn{3}{|c|}{ Main Arm } & \multicolumn{2}{|c|}{ Compensating Arm } \\
\hline & & Link 1 & Link 2 & Payload & Link 1 & Tip mass \\
\hline Mass, kg & 3 & 5.530 & 1.106 & 10 & 3.871 & 5 \\
\hline Length of links, $m$ & - & 1 & 0.2 & - & 0.7 & - \\
\hline Distance of COM from joint, $\mathrm{m}$ & - & 0.5 & 0.1 & - & 0.35 & - \\
\hline Width of links, $\mathrm{m}$ & - & 0.085 & 0.085 & - & 0.085 & - \\
\hline Thickness of links, $\mathrm{m}$ & - & 0.02 & 0.02 & - & 0.02 & - \\
\hline Inclination of pivot of LRM first link with $\mathrm{x}$-axis, $\phi_{0}$, degrees & \multicolumn{6}{|c|}{$70^{0}$} \\
\hline Inclination of pivot of compensating arm with $\mathrm{x}$-axis, $\beta_{1}$, degrees & \multicolumn{6}{|c|}{$110^{0}$} \\
\hline Distance of mounting springs from base centre, $\mathrm{m}$ & \multicolumn{6}{|c|}{$0.2 \mathrm{~m}$} \\
\hline
\end{tabular}

\section{Results and discussion}

In the work [4], the base attitude disturbance was studied through simulation results by considering the model of a three link main arm that is balanced by a similar three link balance arm. However, since reducing the mass of the balance arm will lead to reduced driving torque, the number of links in the balance arm in our work is kept lesser than the main arm. The overall mass reduction is taken as 7.765 $\mathrm{kg}$, of which, $5 \mathrm{~kg}$ due to mass of fluid in the reservoir is dynamically varied. The pitch and bounce motion values obtained by simulating for constant tip mass condition are compared against the corresponding values obtained by varying the tip mass. Thus the effect of varying the tip mass and hence varying the mass of the compensating arrangement is analysed in controlling the base disturbances.
Fig. 10 clearly depicts the suitability of operation of the compensating arm. From the initial condition, the main arm exerts a torque of $25 \mathrm{Nm}$. The compensating arm starts to balance this disturbance. Initially the compensating torque increase from around $-18 \mathrm{Nm}$ upto $-23 \mathrm{Nm}$. This is due to the initial inertia exerted by the compensating arm. However, within 3 seconds, the compensation is achieved. The simulation results verify the compensating model and the hydraulic control unit functions.

Fig. 11 shows the dynamic variation of tip mass of compensating arm. The variation effected is nearly $1.3 \mathrm{~kg}$ (reducing from $4.95 \mathrm{~kg}$ to $3.65 \mathrm{~kg}$ in 3 seconds) as needed for the compensation requirement. It can be ascertained that for larger range of motion of main arm this variation will be greater. 
We further compare the error torque, bounce and pitch of the base for the two conditions of simulation keeping tip mass constant and varying the tip mass. Fig. 12 shows the plot of error torque. When tip mass is varied the error torque has an initial value of $5.5 \mathrm{Nm}$ which drops to zero in 3 seconds. When tip mass is kept constant, the error torque from an initial value of $9 \mathrm{Nm}$ drops to zero in 8.5 seconds. Thus the arrangement of varying the tip mass for compensation is found advantageous.

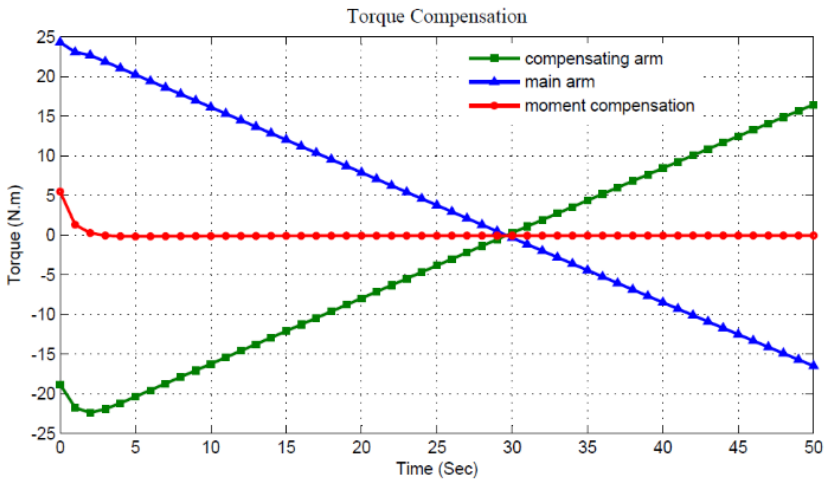

Fig. 10 Torque compensation by actuation of compensating arm

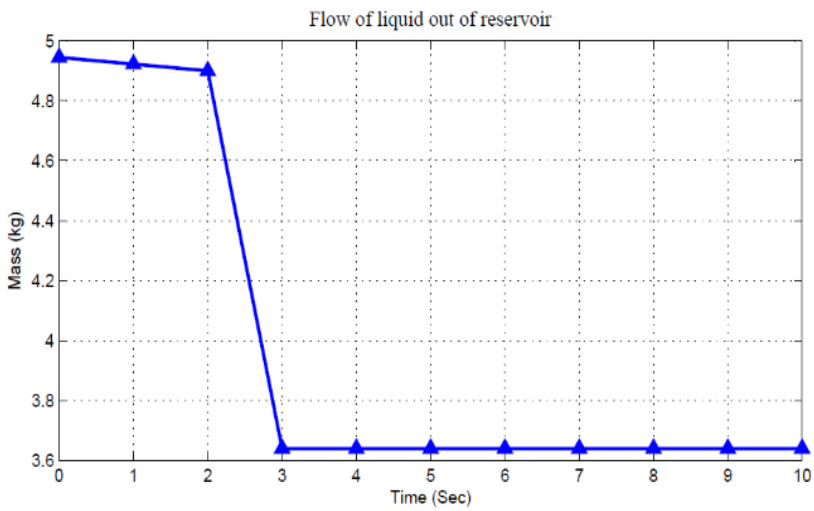

Fig. 11 Tip mass variation in compensating arm

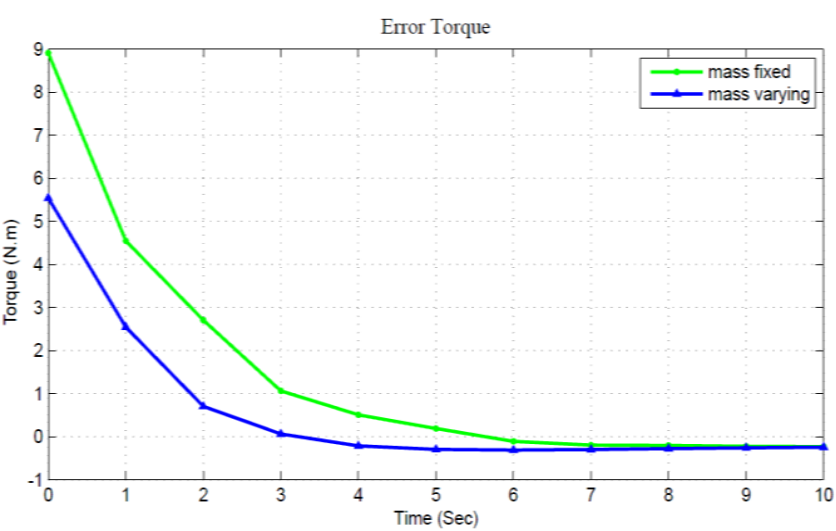

Fig. 12 Error torque between main arm and compensating arm motion

Figs. 13 and 14 show the bounce motion of the base for the cases of varying mass and fixed mass respectively. For the varying mass case, from static equilibrium position, the oscillation of the bounce varies from $-3.58 \mathrm{e}-03 \mathrm{~m}$ to $-3.675 \mathrm{e}-03 \mathrm{~m}$ during the first cycle. with a maximum absolute value of $0.95 \mathrm{e}-04 \mathrm{~m}$. In case of mass being fixed, this maximum value of oscillation is found to be $-3.61 \mathrm{e}-03$ and the oscillation falls to a value of $-3.84 \mathrm{e}-3$ in the first cycle. The maximum absolute value of bounce motion is $2.3 \mathrm{e}-04 \mathrm{~m}$. It is seen that in the mass varying case the oscillations persist, though at a much diminished level till around 7.5 seconds. This might be due to the continuous variation of tip mass in the compensating system. This minor oscillation in the bounce is going to affect only the position of the manipulator and hence may not be detrimental as attitude disturbance.

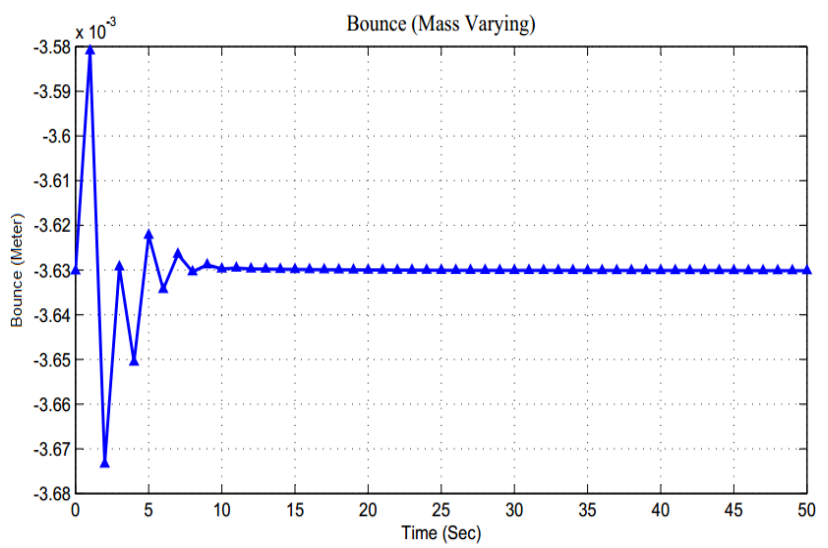

Fig. 13 Bounce motion of base for tip mass varying condition

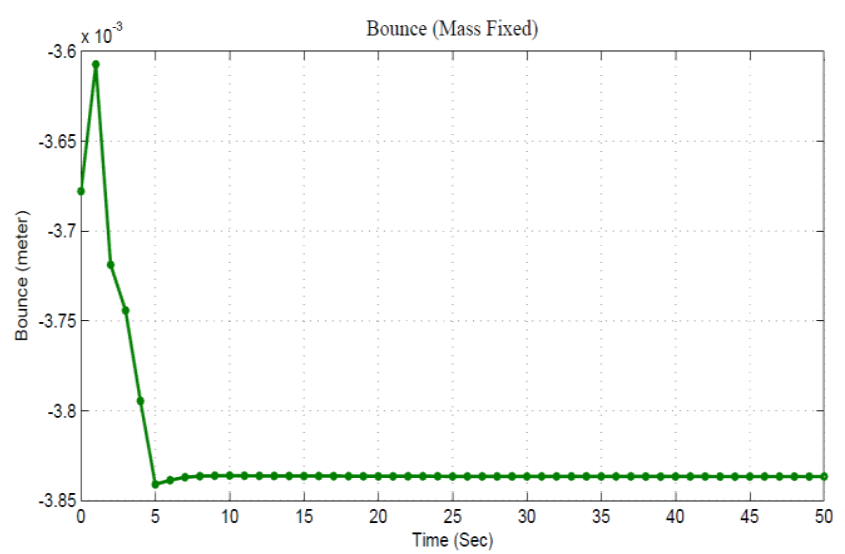

Fig. 14 Bounce motion of base for tip mass fixed condition

The pitching, yaw and roll of the space base is very important in maintaining the proper attitude. For our planar case we have plotted the pitch movement. From Fig. 15 and Fig. 16, the simulation results indicate that the pitching in case of varying mass is a maximum of nearly -0.028 radians while this, in the case of mass being fixed, is nearly -0.068 radians. In both cases the disturbance in the form of pitch motion stabilizes at 5 seconds.

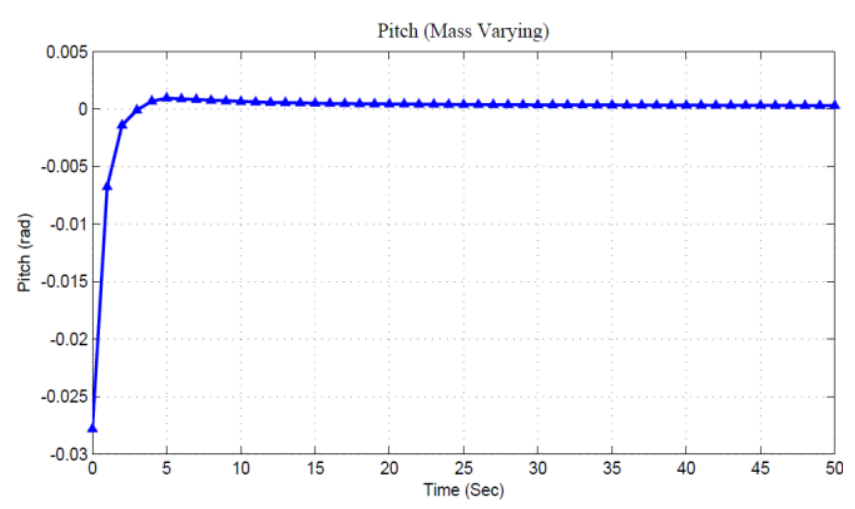

Fig. 15 Pitch motion of base for tip mass varying condition 


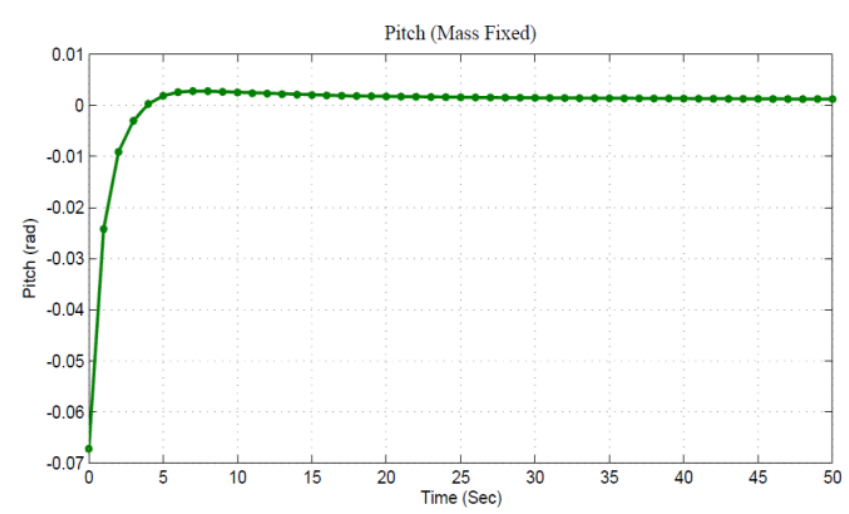

Fig. 16 Pitch motion of base for tip mass fixed condition

Table 2 summarizes the results of the simulation. From the analysis, it is evident that the suggested model of compensating arm arrangement is effectively counteracting the reaction torque at the base. Secondly, it is also evident that the compensating arm arrangement with the provision of varying its mass is better than keeping the mass of the arrangement as fixed in terms of its effectiveness in reducing the base disturbance. This results in better positional accuracy and attitude stability of the LRM system.

\section{Conclusions}

In this work, the method of augmenting balance links to the LRM system for controlling the base disturbances is studied The LRM system was modelled as a two-link manipulator while the balance link was a modelled as a single link compensating arm with lesser mass relative to the LRM model. The provision of a variable tip mass in the compensating arm was suggested and its effect in controlling base disturbance was studied from the simulated values of pitch and bounce of the base.

The simulation results indicate that the suggested compensating arrangement and its control through the hydraulic control circuit is effective in achieving the compensation. The results also suggest that the arrangement of varying the mass of the compensating arm contributes to reduced base disturbances. For this case, it can be stated that a lesser, and slightly varying, drive torque is required, thus addressing the need for economizing on costly space fuel. Hence the concept of incorporating a balance arm with lesser mass and the provision for dynamically varying this mass is a suitable choice for addressing the base disturbances.

Summary of results

Table 2

\begin{tabular}{|c|c|c|c|c|}
\hline $\begin{array}{c}\text { Sl. } \\
\text { No. }\end{array}$ & Metric measured & $\begin{array}{c}\text { Results when tip mass of } \\
\text { compensating arm is varied }\end{array}$ & $\begin{array}{c}\text { Results when tip mass of } \\
\text { compensating arm is fixed }\end{array}$ & $\begin{array}{c}\% \text { lesser in case of } \\
\text { varying mass }\end{array}$ \\
\hline 1 & Error torque (initial value) & 5.5 N.m. (settles at 3 s) & 9 N.m. (settles at 8.5 s) & $38.9 \%$ \\
\hline 2 & Max. absolute value of Base bounce motion, $S$ & $0.95 \mathrm{e}-04 \mathrm{~m}$ & $2.3 \mathrm{e}-04 \mathrm{~m}$ & $58.7 \%$ \\
\hline 3 & $\begin{array}{c}\text { Max. absolute value of } \\
\text { Base pitch motion, } \delta\end{array}$ & 0.028 radians & 0.068 radians & $58.8 \%$ \\
\hline
\end{tabular}

This conceptual method of varying-mass compensating arm can be extended to study the dynamics of LRM for space environment. Also other methods of varying the mass, like incorporating air jets in the compensating arm that send out blasts of calculated amount of air, can be explored and analysed.

\section{References}

1. Di Pippo, S.; Colombina, G.; Boumans, R.; Putz, P. 1998. Future potential applications of robotics for the International Space Station, Robotics and autonomous systems 23(1-2): 37-43.

2. Mavroidis, C.; Dubowsky, S.; Raju, V. 1995. Endpoint control of long reach manipulator systems, Proc. 9th World Congr. IFToMM, pp. 1740-1744.

3. Yoshida, K.; Mavroidis, C.; Dubowsky, S. 1997. Experimental research on impact dynamics of spaceborne manipulator systems, Experimental Robotics IV, pp. 436-447. Springer, Berlin, Heidelberg.

4. Huang, P.; Xu, Y.; Liang, B. 2005. Balance control of multi-arm free-floating space robots during capture operation, Robotics and Biomimetics (ROBIO), IEEE International Conference, pp. 398-403.

5. Dwivedy, S. K.; Eberhard, P. 2006. Dynamic analysis of flexible manipulators, a literature review, Mechanism and machine theory 41(7): 749-777.

6. Dubowsky, S.; Papadopoulos, E. 1993. The kinematics, dynamics, and control of free-flying and free-floating space robotic systems, IEEE Transactions on robotics and automation 9(5): 531-543.

7. Torres, M. A.; Dubowsky, S.; Pisoni, A. C. 1996. Vibration control of deployment structures' long-reach space manipulators: The P-PED method, Robotics and Automation, IEEE International Conference 3: 24982504.

8. Trudnowski, D. J.; Baker, C. P.; Evans, M. S.; 1993. Damping control of a large flexible manipulator through inertial forces of a small manipulator, American Control Conference, pp. 2878-2879.

9. Sharf, I. 1996. Active damping of a large flexible manipulator with a short-reach robot, Journal of dynamic systems, measurement, and control 118(4): 704-713.

10. Liang, B.; Xu, Y.; Bergerman, M. 1997. Dynamically equivalent manipulator for space manipulator system. 1, Robotics and Automation, IEEE International Conference 4: 2765-2770.

11. George, L. E.; Book, W. J. 2003. Inertial vibration damping of a flexible base manipulator, JSME International Journal Series C Mechanical Systems, Machine Elements and Manufacturing 46(3): 798-806.

12. Zhu, S. J.; Zheng, Y. F.; Fu, Y. M. 2004. Analysis of non-linear dynamics of a two-degree-of-freedom vibration system with non-linear damping and nonlinear spring, Journal of Sound and Vibration 271(1-2): 15-24.

13. Chalfoun, J.; Bidard, C.; Keller, D.; Perrot, Y.; Piolain, G. 2007. Design and flexible modeling of a long reach articulated carrier for inspection. In Intelligent 
Robots and Systems, IEEE/RSJ International Conference, pp. 4013-4019.

14. Özer, A.; Semercigil, S. E. 2008. An event-based vibration control for a two-link flexible robotic arm: Numerical and experimental observations, Journal of Sound and Vibration 313(3-5): 375-394.

15. Cocuzza, S.; Pretto, I.; Debei, S. 2010. Reaction torque control of redundant space robotic systems for orbital maintenance and simulated microgravity tests, Acta Astronautica 67(3-4): 285-295.

16. Nenchev, D. N. 2013. Reaction null space of a multibody system with applications in robotics, Mechanical Sciences 4(1): 97-112.

17. Minghe, J. I. N.; Cheng, Z. H. O. U.; Yechao, L. I. U.; Hong, L. I. U. 2017. Analysis of reaction torque-based control of a redundant free-floating space robot, Chinese Journal of Aeronautics 30(5): 1765-1776.

18. van der Wijk, V.; Herder, J. L.; Demeulenaere, B. 2009. Comparison of various dynamic balancing principles regarding additional mass and additional inertia, Journal of mechanisms and robotics 1(4).

19. Gardner, J. F. 2001. Simulations of machines using Matlab and Simulink, Cengage Learning India Edition.
A. Srinivasan, A. Jegan

SIMULATION STUDY OF BALANCING ARM

ARRANGEMENT FOR CONTROLLING BASE

DISTURBANCES IN LONG REACH MANIPULATORS

S u m m a r y

The dynamics of the Long Reach Manipulators create disturbances in its supporting base structure. This causes positional and attitude inaccuracies of the endeffector, thwarting the accomplishment of the desired mission task. Hence the base disturbance problem has been a subject of research since long time bringing solutions from varied domains. This paper analyzes the balance link concept for compensating the disturbances. The LRM system is modelled as a two-link serial chain with payload, and the balance arm, as a single link with tip mass that is dynamically varied by controlling the quantity of the hydraulic fluid in the bellows-like reservoir at the tip. The supporting base is modelled as a half car. Simultaneous constraint matrices are set up for the arrangement, and dynamic simulation is performed using SIMULINK. The pitch and bounce motions of the base are studied along with the torque error during compensation. The simulation plots are obtained for two conditions of compensating arm with fixed tip mass and with varying tip mass. The results confirm the suitability of the suggested compensating arrangement in controlling base disturbances.

Keywords: balance link, base disturbance, compensating arm, long reach manipulator, variable tip mass, simultaneous constraint method.

Received August 14, 2020 Accepted June 02, 2021

This article is an Open Access article distributed under the terms and conditions of the Creative Commons Attribution 4.0 (CC BY 4.0) License (http://creativecommons.org/licenses/by/4.0/). 